Available online on 15.03.2018 at http://jddtonline.info
Journal of Drug Delivery and Therapeutics
Open Access to Pharmaceutical and Medical Research
$\begin{gathered}\text { (0) 2011-18, publisher and licensee JDDT, This is an Open Access article which permits unrestricted non- } \\ \text { commercial use, provided the original work is properly cited }\end{gathered}$

Open $\odot$ Access

Research Article

\title{
ESTROGEN ANCHORED NANOMICELLES FOR COMBINATIONAL DRUG DELIVERY WITH ENHANCED THERAPEUTIC EFFICACY: A PROTEOMICS GUIDED INVESTIGATION FOR MULTIFUNCTIONAL NANOTHERAPEUTICS
}

\author{
Juan-Juan Yin ${ }^{1,2^{*}}$, Christopher Burgess ${ }^{2}$, Liping Shu ${ }^{3}$, Stepan P. Shumyak ${ }^{2}$, Nicholas Raymond ${ }^{2}$, Li Xu$^{4}$, \\ Lu-Lin $\mathrm{Li}^{1}$, Shu-Ming $\mathrm{He}^{1}$ \\ ${ }^{1}$ Xiaolan Hospital, Southern Medical University, ZhongShan, Guangdong 528415, China \\ ${ }^{2}$ Department of Pharmaceutical Sciences, College of Pharmacy, University of South Florida, Tampa, Florida, 33612, USA \\ ${ }^{3}$ Guizhou Provincial Key Laboratory for Regenerative Medicine, Stem Cell and Tissue Engineering Research Center \& Sino-US \\ Joint Laboratory for Medical Sciences, Guiyang Medical University, Guiyang, Guizhou 550004, China \\ ${ }^{4}$ Ji-Nan Central Hospital, Ji-Nan, ShangDong 250013, China
}

\section{ABSTRACT}

The employment of the stable isotope labeling with amino acids in cell culture (SILAC) based proteomic analysis as a guidance tool for investigation of cellular response of a multifunctional nanodelivery system has been described for cancer therapy. The multifunctional nanodelivery system in this study was based on an estrogen anchored multimodal nanomicelle (NPG). The key components of the multifunctional nanomicelle consist: $\beta$-cyclodextrin conjugated estrone (CDE1), an escort molecule; polymeric BH3 mimetic, a proapoptotic BcL-2 inhibitor; and the mitotic catastrophe agent paclitaxel. They were assembled into the nanomicelle by multiple weak interactions including hydrophobic/hydrophilic interaction and host-guest recognition. The resulting nanomicelle exhibited unimodal morphology with average size of $170 \mathrm{~nm}$ with sustained release. It was found that the nanoparticle exhibits excellent anti-tumor activities for the treatment of breast adenocarcinoma with exceptional targeting efficacy both in MCF-7 tumor bearing mice. NPG as a new drug delivery system demonstrated several merits such as the increased drug uptake in breast tumor tissue, low toxicity, potent tumor growth retardation and metastasis inhibition, as well as potential clinical practicality without compromising liver, kidney and immune function and ameliorating the conventional chemotherapeutics induced phlebitis in breast tumor bearing nude mice model. The systematic SILAC based proteomics study and the subsequent validation revealed that the synergistic induction of mitotic catastrophe through enhanced G2/M phase arrest and PI3K/Akt/mTOR mediated autophagy, account for the exceedingly potent anti-tumor activity of this convergent nanomicelle. Additionally, the verification of the top upregulated gene from the proteomics profiling revealed that the overexpression of zinc finger protein 350 (ZNF350/ZBRK1) is associated with the enhanced antitumor effect induced by NPG.

Keywords: Drug targeting, Estrogen Receptor, Proteomics, Paclitaxel, Gossypol, Synergism

Article Info: Received 09 Jan, 2018; Review Completed 16 Feb, 2018; Accepted 16 Feb, 2018; Available online 15 March, 2018

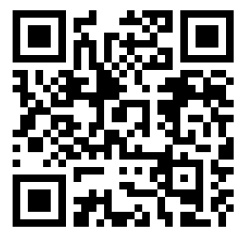

Cite this article as:

Yin JJ, Burgess C, Shu L, Shumyak SP, Raymond N, Xu L, Li LL, He SM, Estrogen anchored nanomicelles for combinational drug delivery with enhanced therapeutic efficacy: a proteomics guided investigation for multifunctional nanotherapeutics, Journal of Drug Delivery and Therapeutics. 2018; 8(2):41-53

DOI: http://dx.doi.org/10.22270/jddt.v8i2.1662

*Address for Correspondence

Juan-Juan Yin, Xiaolan Hospital, Southern Medical University, ZhongShan, Guangdong 528415, China 


\section{INTRODUCTION}

The heterogeneity and instability of cancer genomes results in highly variable clinical outcomes for cancer treatment. ${ }^{1}$ In current clinical practice, combination regimens are routinely applied although the scientific evidence for the combination therapies is weak. In a hope of improving the clinical outcome of the combination regimens, combined modality therapy in a nanoformulation in terms of multifunctional nanomedicine has attracted increasing attention and provides a promising direction for cancer therapy. ${ }^{2}$ Multifunctional nanomedicines integrate comprehensive functionalities with different mechanisms of drug action, providing targeted, ratiometric delivery of drugs or genes, consequently making significant improvement of their pharmacokinetic/dynamic profile compared to the conventional nanomedicines. ${ }^{3-6}$ However, application of the majority of multifunctional nanodelivery systems are substantially hampered due to their inherent complexity in vivo, including the heterogeneities of tumor vascular permeability, $^{7}$ varying pharmacokinetics, nonspecific biodistributions, and unpredicted adverse effects. One critical reason for the limitations is the lack of understanding of the sophisticated drug actions amongst the multiple constituents in the integrated nanoparticles for the treatment of cancer.

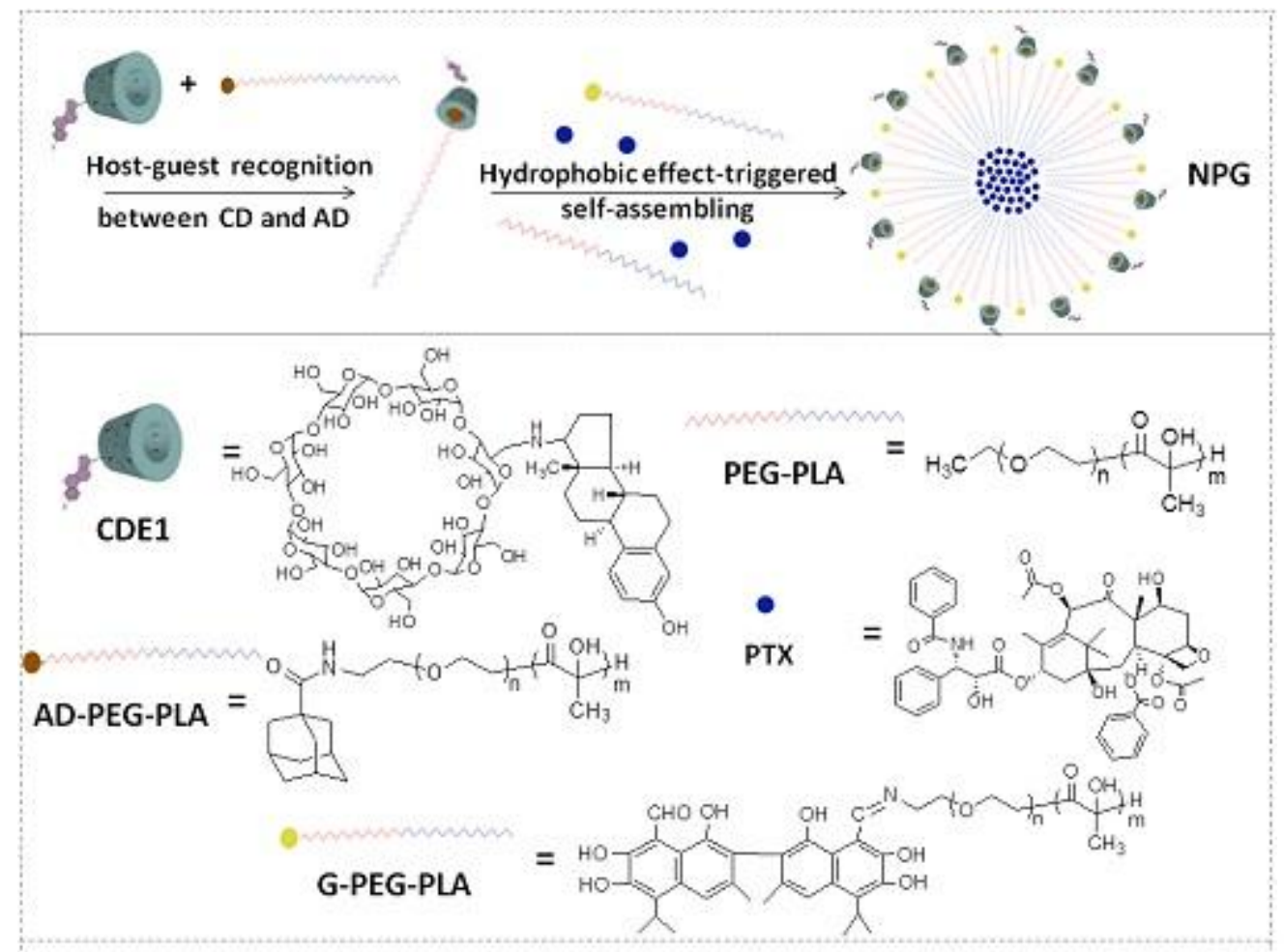

Figure 1: Schematic of the novel nanoparticle. Above is a schematic representation of nanoparticles developed in this study. Targeted multifunctional Nanoparticles formulated by functionalized PEG-PLA and synergetic chemotherapeutics, for improved anticancer activity. The various modifications to the PEG-PLA polymer allow the nanoparticle to be capable of targeted co-delivery of therapeutics. (PEG-PLA: copolymers of polyethylene glycol and polylactic acid; CD: cyclodextrin; AD: Adamantyl group)

Previous approaches for assessing drug synergy in multifunctional nanoparticles were usually empirical which always suffered from the low accuracy and uncertain predictability. ${ }^{6}$ No doubt, the accurate information of proteome wide cellular response triggered by the multifunctional nanoparticle from proteomics would provide a key to understanding the molecular mechanism underlying the anti-tumor effects from the multiagents interactions in the highly convergent nanoparticles, and consequently facilitate the development of new generation nanotherapeutics for cancer. Proteomics reveals large-scale information of proteomes and uncovers important points in the cell signaling from complex mixtures; and they have attracted increasing attention in drug discovery. ${ }^{8-9}$ SILAC based proteomics, which relies on the incorporation of amino acids with substituted stable isotopic nuclei and enables data interpretation and quantification more accurate and robust, has emerged as a powerful approach in pharmaceutical sciencej. However, the proteomics has never been demonstrated in the investigation of the multifunctional nanoparticle triggered cellular pathways for cancer therapy..$^{10-11}$ Proteomics facilitated analysis of cellular trafficking induced by nanoparticles would be particularly attractive because it could reveal the mechanic paradigms of the anti-tumor effects from a complex high convergent nanoparticle, consequently, facilitating the development of new drugs for treatment of cancers. We expect that the marriage of SILAC based proteomics and multifunctional nanoparticle would open an unprecedented door for cancer research. 
Herein, we present the first example of the cancer therapeutic study for the combinational therapeutics in targeting nanomicelles as drug delivery systems guided by proteomics. The judicious design and synthesis of a multifunctional polymer micelle which incorporates functionalities including membrane estrogen receptor (mER) targeting, mitotic catastrophe inducing, and BcL2/Bcl-xL inhibiting for mER (+) breast adenocarcinoma is depicted in Figure 1. In addition to the evaluation of the antitumor effect in vitro and in vivo, the molecular mechanism behind the therapeutic outcome was investigated by systematic epigenetic study and SILAC based proteomes mining. The top molecules and top pathways in the response of the nanoparticle NPG were analyzed. The anti-tumor potency of NPG results from synergistically enhanced mitotic G2/M phase arrest and autophagy instead of the expected apoptosis. The validation of the top gene from the proteomics profiling revealed that the upregulation of ZNF350 is associated with the enhanced antitumor effect of NPG and represents a potential therapeutic target induced by the nanoparticles.

\section{RESULTS AND DISCUSSION}

Design, Synthesis and Characterization of the Multifunctional Nanoparticle

The selection of an appropriate ligand for a nanoparticle is critical to ensure its precise and exclusive tumor targeting for the designed nanoparticle based on different types of cancers. To initiate our proteomic directed cancer therapeutic study of multifunctional nanoparticles, $\operatorname{mER}(+)$ breast cancer was utilized as a model to test our concept. Thus estrogen was selected as a targeting vector since mERs on the breast cancer cell membrane such as classic ER $\alpha, E R \alpha$ splicing variants (36 kDa, $46 \mathrm{kDa}$ etc.), ${ }^{12-14}$ ER-X, ${ }^{15}$ GPR30, and Gaq$\mathrm{mER}^{16}$ could bind by estrogens. On the other hand, the dual drugs functioned in different mechanisms such as the anti-mitotic agent paclitaxel $(\mathrm{PTX})^{17}$ and a BH3 mimetic gossypol $(\mathrm{Gsp})^{18}$ were encapsulated in the nanoparticle. With these key elements synthesized, we fabricate a multifunctional nanodrug delivery system through host-guest recognition between cyclodextrins and adamantyl groups based on our previous established approaches 19 and hydrophobic drug interaction triggered self assembling. ${ }^{20}$

In particular, the estrone anchored multifunctional nanomicelles (NPG) consists of five components as shown in Figure 1 and Scheme S1 in Supp Info. They are: anti-mitotic agent paclitaxel (PTX), which was encapsulated in the inner core of NPG; natural BH3 mimetic, a racemic Gsp with low aqueous solubility as adjuvant drug conjugated to the terminal of the di-block polymer to form the hydrophilic G-PEG-PLA; CDE1 as the targeting moiety; adamantane attached polymer ADPEG-PLA as linker of CDE1 to the nanoparticle; PEGPLA as the base material in NPG.

The synthetic procedure for the NPG was convergent and straightforward. First, CDE1 was readily synthesized from estrone and amino- $\beta$-cyclodextrin ${ }^{19}$ through Borch reduction as a targeting moiety. The polymer linker AD-PEG-PLA was prepared by an amidation reaction of the corresponding commercially available admantyl carbonyl chloride and the terminal amine derived PEG-PLA. The drug G-PEG-PLA was installed through the formation of imine functionality from the gossypol and amino-PEG. Through the wellknown host-guest interactions of admantyl group and the cyclodextrin functionality, the conjugate of CDE1 and AD-PEG-PLA was readily formed. After all the components functionalized, the final multifunctional nanoparticle (NPG) was fabricated by a hydrophobic drug-triggered self-assembly and host-guest recognition with overall hydrophilic property. The structure of, the resultant nanoparticle and the corresponding intermediates were fully characterized by nuclear magnet resonance (NMR), mass spectrometry (MS), fourier transform infrared spectroscopy (FTIR) and liquid chromatography (LC) as shown in Figure S1-3.

To determine the morphology, particle size distribution and surface zeta-potential of the resulting multifunctional NPG, a variety of techniques such as scanning electron microscopy (SEM), transmission electron microscopy (TEM) and dynamic light scattering (DLS) were carried out. The NPG shows a unimodal and narrower size distribution of $18 \%$. The SEM image in Fig 2A showed spherical particles with an average diameter of approximately $170 \mathrm{~nm}$. The size was twice that of the starting material PEG-PLA (Fig S7), indicating the geometrical fabrication of the nanoparticle. TEM microscopic images of NPG displayed an obvious morphology change compared to that of the starting polymer, again indicating the occurrence of the self-aggregation. Additionally, the TEM image clearly showed that the nanoparticle consists of multiple small spherical domains. The small domains were around $170 \mathrm{~nm}$ according to the statistical analysis of three samples, supporting that the small domains were conventional micelles self-assembled from the subunits. In addition, the surface charge of the NPG was investigated by DLS, and the results were consistent TEM and SEM, in addition, it showed that the $\zeta$-potential value is $5.8 \mathrm{mV}$, suggesting thermodynamic and kinetic stability of the nanoparticle. Thus, the asprepared nanoparticles were formed through a selfassembly by host-guest recognition and hydrophilic interactions. 

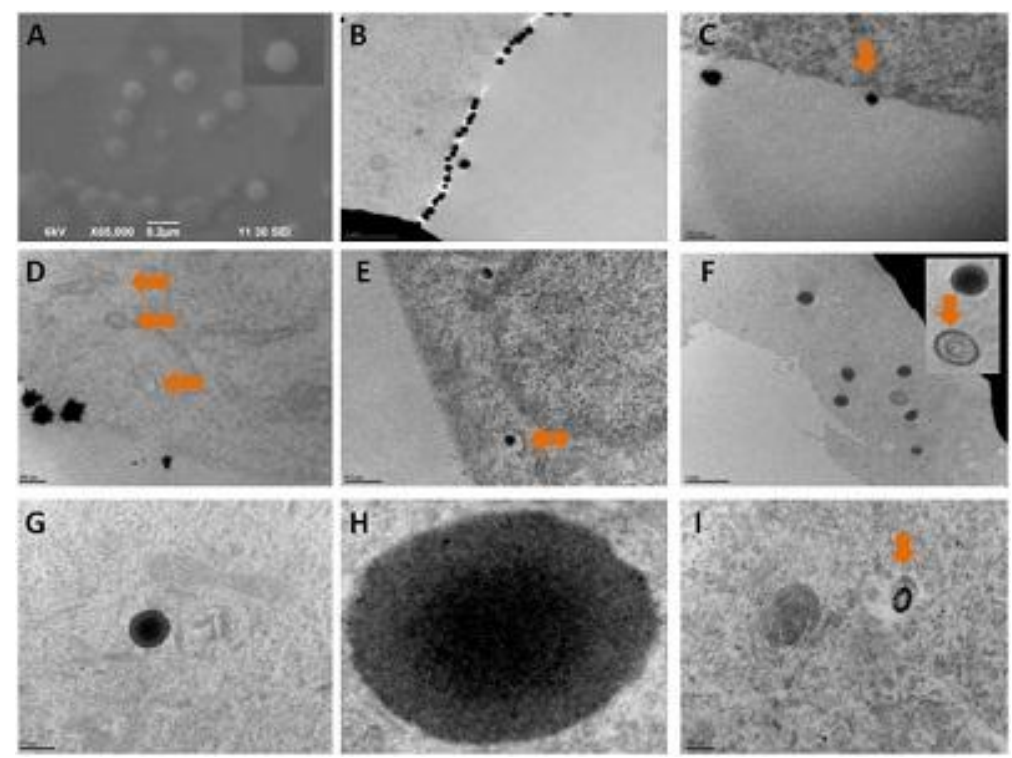

Figure 2: Transmission electron microscopic and scanning electron microscopic observations of the multi-functional polymeric micelles (A) SEM images of NPG (65K); (B-D) TEM images of NPG, samples were prepared from the MCF-7 cells after NPG exposure for $5 \mathrm{~min}$; B: The NPG nanoparticles have been observed line alone the cell membrane (10k); C: Endocytosis of the nanoparticle (80k); D: Vesicles (as arrows shown) migrate actively to the cell membrane to internalize the nanoparticle (150k). (E) Samples were prepared from the MCF-7 cells after NPG exposure for $10 \mathrm{~min}$, vesicles break apart as the arrow shown (40K). (F-I) Samples were prepared from the MCF-7 cells after NPG exposure for $15 \mathrm{~min}$; F: Nanoparticles are shown inside the cells, some of the nanoparticles have been digested, leave the empty out shell (as the arrow shown); G: NPG (with averaged diameter of $170 \mathrm{~nm}$ ) are clearly shown the outer shell and inner core with drug encapsulated (40k); H: One enlarged nanoparticle of NPG; I: The encapsulated drug released and dispersed inside the MCF-7 cells as the arrow shows.

Moreover, the morphology of the nanoparticle in MCF7 cancer cell was investigated by TEM. TEM images in tissue also showed the structure of the nanoparticle with the diameter of average $170 \mathrm{~nm}$ outer shell and inner core with drug encapsulated. Importantly, excellent cell permeability and drug release capability of the multifunctional nanoparticle was observed. As shown in Fig 2F, the TEM image showed that there were quite a few nanoparticles inside the cells, while some of the nanoparticles had been digested and left the out shell alone. Notably, at $15 \mathrm{~min}$ after the nanoparticles were internalized into the tumor cells, the typical and delicate two-phase micelle structure with outer hydrophilic layers and inner hydrophobic cores has been clearly observed (Figure 2F-H), the enlarged one polymeric micelle is shown in Figure $2 \mathrm{H}$, which also demonstrate the successful preparation. The encapsulated drug was released and dispersed inside the MCF-7 cancer cells as shown in Fig 2I. These results demonstrated the promising prerequisite of the resulting multifunctional nanoparticles in treatment of cancer.

Additionally, the NPG demonstrated excellent ratiometric drugs release capability and low cytotoxicity compared to the parent drugs. For instance, the drug release from the nanoparticle significantly lags behind the parent drug, its release was continuous and sustained up to $16.8 \%$ for the nanoparticle with $k d$ value of 20.38 vs $83.9 \%$ for the encapsulated fluorescent probe with $k d$ $=0.4$ after $56 \mathrm{~h}$, the drug in the nanoparticle was abruptly dissociated within $0.5 \mathrm{~h}$ and then slightly release while the parent drug has a sharp rise at 8 and 24 $\mathrm{h}$ with relative drug release percentage of $17.8 \%$ and $51.9 \%$ respectively (Figure S5). As for the cytotoxic assays, NPG shows a significantly lowered IC50 value (1.9 $\mathrm{nM})$ than the corresponding nontargeting nanopaticle NPC $(3.9 \mathrm{nM})$. Additionally, the progressively declined cytotoxicity response and the long-term drug release of the NPG were more predominant up to $72 \mathrm{~h}$ compared with PTX due to the stability of the NPG.

Moreover, the NPG showed the most potent tumor sphere inhibition compared to blank, Gsp, G-PEG-PLA, NPC, and PTX groups as demonstrated in the ex vivo tumor colony inhibition study (Figure S10). For example, when the tumor colonies were exposed to the drugs for $48 \mathrm{~h}$, the tumor colony at the NPG treated group exhibited a $44.0 \%$ decrease in size, while only $8.34 \%$ in G-PEG-PLA treated group and $18.43 \%$ for PTX treated group.

The membrane receptor mediated endocytosis procedure during the internalization of NPG by mER (+) breast cancer cells has been proposed due to the targeting function of CDE1 interpolated in NPG and verified based on the findings. Firstly, TEM was used to capture the process at increasing time periods after exposure to NPG at $50 \mathrm{nM}$ in MCF-7 cells. NPG can only be observed lined up along the cell membrane at the $5 \mathrm{~min}$ time point after the treatment with NPG (Figure 2B, S7), such tight junctions between the nanoparticles and the tumor cell membrane indicates that NPG bonds with plasma membrane protein(s) or certain receptor(s), otherwise the physical association between NPG and cell surface would be disrupted and rinsed off by the following procedure before fixation. The caveolae-like pit with a nanoparticle can be observed on the cell membrane in Figure 2C. During the internalization, the 
newly formed round and small vesicles migrate actively to the cytoplasma membrane for the uptake of the nanoparticles as the arrows show in Figure 2D. At a later time point of $10 \mathrm{~min}$, endosomal vesicles have been observed to disperse and release the nanoparticle drug after internalization as arrows show in Figure 2E. These results provide evidence for the membrane receptor mediated endocytosis for NPG uptake which took place in a quick process. Cristae of mitochondria and other healthy organelles can be observed (Figure S7) indicating the undamaged cell stage within short drug exposure time. The delicate microstructure of the released drugs and empty polymer shells from the polymer micelles have been captured during the drug release and digestion which give a clear picture for the dynamic process during the internalization, drug release and digestion of NPG by the tumor cells. Secondly, to further confirm the interaction of NPG with mER, flow cytometry analysis was performed in MCF-7 cells. The compound Ada-DOX ${ }^{19}$ with intrinsic fluorescence was encapsulated in the estrogen anchored nanoparticles instead of PTX as same equivalent (Figure S19). Figure S19A shows representative quantitative flow cytometric results of the cellular uptake of the estrogen anchored nanopaticles (T) and non-targeting nanoparticles (N) without estrogen moiety attached. Within $18 \mathrm{~h}$ of drug exposure, estrogen anchored nanoparticles uptake by
MCF-7 cells has shown a tendency to increase over nontargeting nanoparticles, and considerably augmented uptakes by $11.8 \%$ and $34.6 \%$ observed at time point of 24 and $48 \mathrm{~h}$ compared with non-targeting nanoparticles, which indicates that mER facilitate the uptake of the targeting nanoparticles over the nanoparticle without the estrogen ligands attached. At last, we performed the competition assay with NPG using the synthetic ER ligand analog ethinyl estradiol (EE) and selective ER modulator tamoxifen (TAM) in MCF-7 cells. The results (Figure S19B) showed that both EE and TAM at 0.1 and $1 \mu \mathrm{M}$ inhibited NPG uptake in a concentration independent manner in MCF-7 cells. EE and TAM diminished the uptake of nanoparticles by $25.7 \%(1 \mu \mathrm{M}$

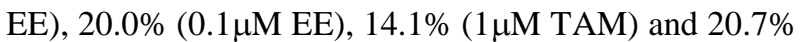
$(0.1 \mu \mathrm{M}$ TAM) respectively in MCF-7 cells (***P < 0.001). Based on the findings, the mER mediated endocytosis of NPG internalization has been confirmed.

\section{SILAC Based Proteomic Quantification Analysis}

Initial results have shown that the multifunctional NPG exhibited excellent tumor cell growth inhibition, and sustained drugs release through receptor mediated endocytosis. In order to gain comprehensive mechanic information for understanding of cell response triggered by NPG in breast carcinoma cells, a systematic proteomic study of NPG was conducted.
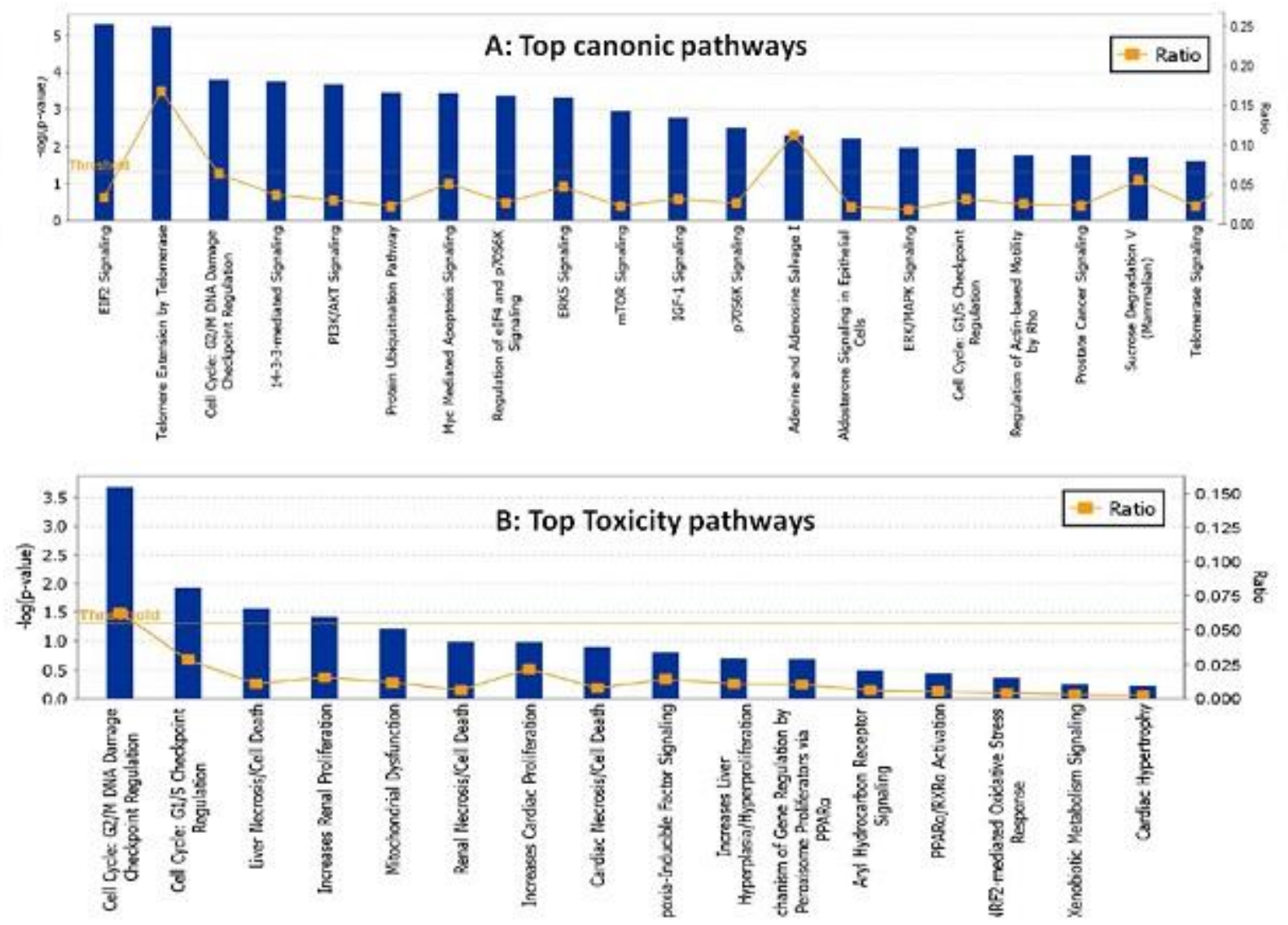

Figure 3 Top canonical pathways (A) and top toxicity pathways (B) enriched in the proteome of multifunctional nanoparticles NPG treated breast cancer cells generated using IPA software. Orange dots indicate ratio, ratio was calculated by dividing the number of genes from our dataset that map to each single pathway by the total number of genes included into the canonical and toxicity pathways. Blue bars denote - log (P-value) by right-tailed Fisher's Exact test, indicating the statistical significance of the molecules in each criterion. Ratio and -log (P-value) are indicated on the left and right side of the histogram, respectively. 
Specifically, stable isotope labeling with amino acids in cell culture (SILAC) based quantitative proteomic analysis has been performed, the entire proteome of drug treated MCF-7 cells is metabolically labeled by heavy, non-radioactive isotopic variants of amino acids equally combined with unlabeled samples thus making it distinguishable by nanoLC-MS/MS analysis. Cell lysates were prepared right after drug treatment of NPG, Gsp, G-PEG-PLA, NPC and PTX on MCF-7 cells for 12 $\mathrm{h}$ at $50 \mathrm{nM}$, and then the cell lysates were proteolytically digested and desalted. The protein expression altered in MCF-7 breast cancer cells treated with NPG compared with its constituents: PTX, Gsp, G-PEG-PLA and NPC.
We obtained a data set of 138 pro teins for NPG, 315 proteins for Gsp, 294 proteins for G-PEG-PLA, 495 proteins for NPC and 301 proteins for PTX (Addendum I) after filtering the proteins that showed a significant similarity(Pearson's correlation $>0.2$ ).

Top canonical pathways and top toxicity pathways enriched in the proteome of NPG treated breast cancer cells generated using the Ingenuity Pathway Analysis (IPA) software are given in Figure 3. The key pathway network of NPG response in MCF-7 cells algorithmically generated based on their functional and biological connectivity, is graphically represented in Figure 4.

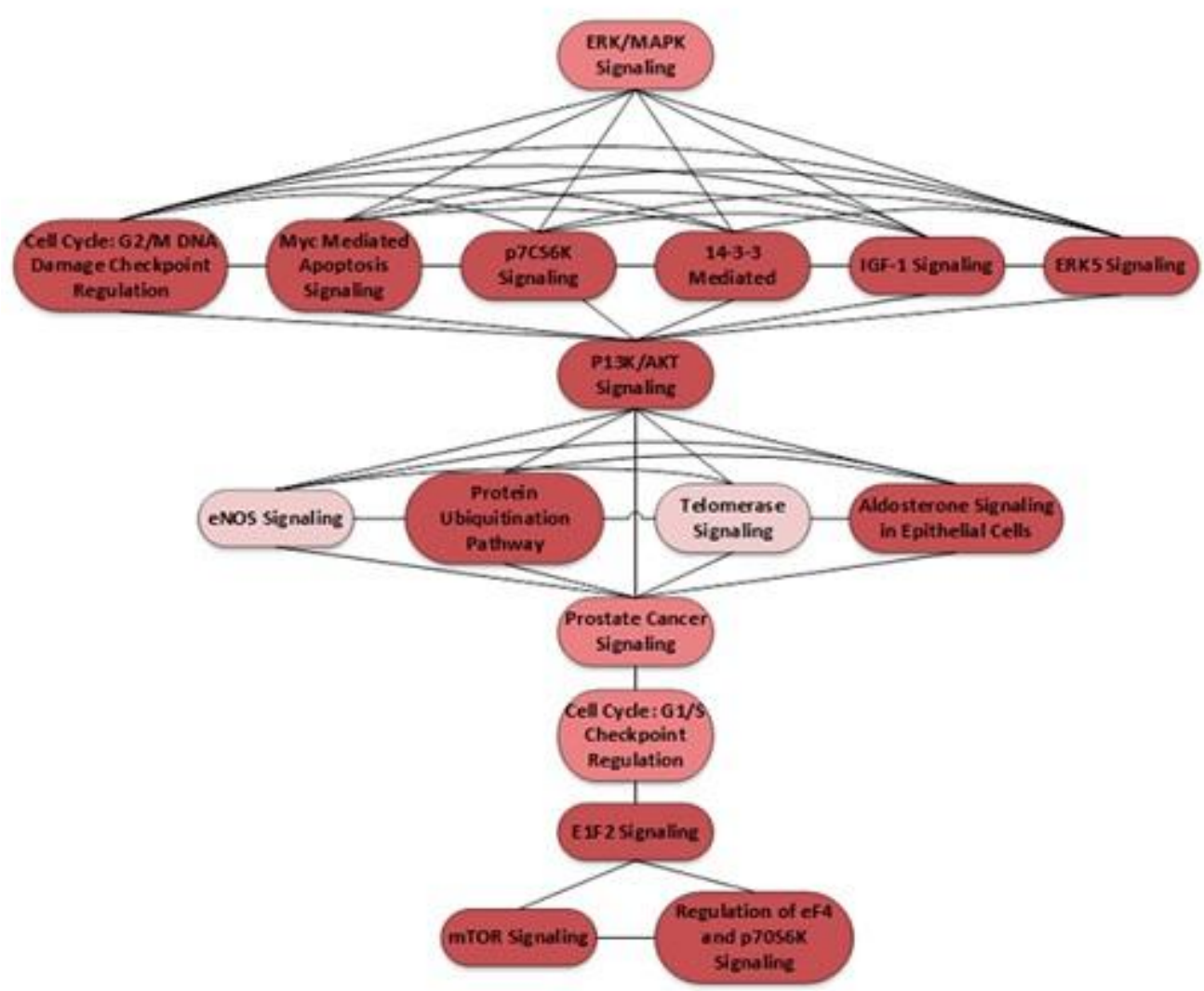

Figure 4: Relationships between highest scored regulated pathways trigger by NPG from the SILAC proteomics in MCF-7 cells analyzed by IPA, algorithmically generated based on their functional and biological connectivity, was graphically represented as boxes (pathways) and lines (the biological relationship between the pathways).

The most significant canonic pathways seen are the cell cycle of G2/M, DNA damage checkpoint regulation signaling, PI3K/AKT signaling, Myc mediated apoptosis, mTOR signaling, ERK5 signaling, telomerase extension signaling constitute the majority of pathways highlighted in the drug response induced by NPG. The cell cycle of G2/M DNA damage checkpoint regulation signaling and $\mathrm{G} 1 / \mathrm{S}$ checkpoint regulation signaling pathways are indicated to play critical roles for the cytotoxicity in response to the drug NPG. Moreover, in the analysis of upstream regulators, it shows the predicted activation of $\beta$-estrodiol relate to the NPG stimulating pathway which reflects the estrogen-like activity of CDE1 (Figure S11 and Addendum II). In the IPA functional analysis, the protein identifications of top up regulated and down regulated molecules and their fold-change expression with respect to NPG treated
MCF-7 breast cancer cells are shown in Table 1 . Notably, among the top genes (or proteins) up regulated and down regulated based on the IPA network analysis, ZNF350 appears to be the most outstanding up regulated gene with 15 fold change unique to $N P G$ group which is absent in Gsp, G-PEG-PLA, NPC and PTX treated groups. In the disease and biofunction examination of proliferation for tumor cell lines (Figure S12), the essential role of ZNF350 is annotated for the MCF-7 cells after NPG treatment. Upregulation of ZNF350 is confirmed after NPG treatment in MCF-7 bearing nude mice and will be discussed. ZNF350 protein has been reported to decreases malignant growth of Hela cells in cell culture and nude mice, and acts as a metastatic suppressor by directly regulating MMP9 in cervical cancer. ${ }^{21}$ 
Table 1 Ingenuity Pathway Analysis (IPA) functional analysis. Annotations for the top ten up- and down-regulated genes are indicated

(A) Top molecules up-regulated

\begin{tabular}{|c|c|c|c|}
\hline Protein ID & Gene Symbols & Genename & Fold change \\
\hline Q9GZX5 & ZNF350 & Zinc finger protein 350 & 15.010 \\
\hline P62805 & HIST1H4A & Histone $\mathrm{H} 4$ & 4.2282 \\
\hline P62913 & RPL11 & 60 s ribosomal protein L11 & 2.6095 \\
\hline P38919 & EIF $4 A 3$ & Eukaryotic initiation factor 4A-III & 2.2024 \\
\hline Q9BY12 & SCAPER & $\begin{array}{l}\text { S phase cyclin A-associated protein in } \\
\text { the endoplasmic reticulum }\end{array}$ & $\begin{array}{l}1.6961 \\
1.6706\end{array}$ \\
\hline P08195-4 & SLC $3 A 2$ & 4F2 cell-surface antigen heavy chain & 1.4003 \\
\hline P05141 & SLC25A5 & ADPIATP translocase 2 & 1.3953 \\
\hline Q9UFE4 & $\operatorname{CCDC} 39$ & Coiled-coil domain-containingprotein & 1.3544 \\
\hline P68104 & EEF1A1 & 39 & 1.285 \\
\hline E9PK25 & CFL1 & Elongationfactor 1-alpha & \\
\hline
\end{tabular}

(B) Top molecules down-regulated

\begin{tabular}{lccc}
\hline Protein ID & Gene Symbols & Gene name & Fold change \\
\hline BSMCJ9 & TRIM66 & Tripartite motif-containingprotein 66 & 0.1246 \\
P13640 & MT1G & Metallothionein-1G & 0.2264 \\
Q62TR5 & CXorf22 & Uncharacterized protein CXorf22 & 0.3276 \\
P16401 & HIST1H1B & Histone H1.5 & 0.3755 \\
P80723 & BASP1 & Brain acid soluble protein 1 & 0.4077 \\
\hline
\end{tabular}

The interaction of ZNF350 with MMP9, BRCA1 $1^{22}$ and microRNAs are also predicted to be a network based on evidence in literature which is shown in Figure S13. The proteomics profiling provided valuable information on mechanism and target druggability that will accelerate the downstream drug development, ${ }^{23-24}$ furthermore, allowing us to achieve precise design and implementation for nanomedicines.

Validation of the Cytotoxic Pathways and the Top Regulated Gene in Vitro and in Vivo from Proteomic Annotation

Annotated by the proteomics studies, we further validated the major pathways for the proteomics profiling such as $\mathrm{G} 2 / \mathrm{M}$ check point regulation, G1/S check point regulation for cell cycle, apoptosis signaling, $\mathrm{PI} 3 \mathrm{k} / \mathrm{AKT} / \mathrm{mTOR}$ mediated autophagy signaling, and Erk/MAPK signaling annotated in Figure 3 and 4. Firstly, the time course effect of the nano-formulations on cell cycle progression was examined to validatethe key network of NPG response in MCF-7 cells (Figure 5 and S15-17). Treatment of the MCF-7 cells with NPG at concentrations of $50 \mathrm{nM}$ led to a significant increase in the $\mathrm{G} 2 / \mathrm{M}$ population over the $2,4,8,12,24,48$ and $72 \mathrm{~h}$ time periods in comparison to PTX, followed by an increased fraction of the sub-G1 population and appearance of tetraploidy. The G2/M population percentage after NPG treatment for $12 \mathrm{~h}$ is $77.6 \%$, $71.03 \%$ and $44.80 \%$ for NPC and PTX. Increasing the length of drug treatment to $72 \mathrm{~h}$ did not further contribute to the $\mathrm{G} 2 / \mathrm{M}$ population increase. Overall, NPG caused the most enhanced G2/M accumulation in MCF-7 cells and the effect sustained for the longest observed time period. NPC shows less effect on cell population change of cell cycle than NPG, while more potent than PTX. In regards to Gsp and G-PEG-PLA treated groups, a slight increase for $\mathrm{G} 2 / \mathrm{M}$ phase was recorded after $12 \mathrm{~h}$ but then change back and became comparable to the control groups for all G1, S, and $\mathrm{G} 2 / \mathrm{M}$ phase population. In the investigation of cell cycle associated proteins by time course western blot (WB) for
2 to $72 \mathrm{~h}$ drug treatments, the G2/M phase checkpoint regulation though the p53 dependent and $221^{\mathrm{WAF} 1 / \mathrm{CIP} 1}$ associated CDK1/Cyclin B1 inhibition pathway was shown to be the predominate mechanism to induce tumor cell death induced by NPG. Additionally, MCF-7 cells underwent morphological changes from a polygonal appearance to rounded shape in response to NPG, NPC and PTX treatments at $50 \mathrm{nM}$ for $12 \mathrm{~h}, \mathrm{NPG}$ and NPC caused more cells damage compared with PTX, while the cells have no obvious morphological difference after the Gsp and G-PEG-PLA treatment at 50 $\mathrm{nM}$ for $12 \mathrm{~h}$ (Figure 5D).

Moreover, mTOR signaling is within the top ten pathways indicated in the proteomics profile about MCF-7 breast cancer cells response after NPG treatment. The time course of NPG preferentially induces mTOR dependent autophagy in MCF-7 cells and in MCF-7 tumor bearing nude mice, was monitored by flow cytometry, WB and confocal microscopy (Figure 6 and S18). Autophagosome vacuole formation and protein light chain 3 (LC3I/II) upregulation were observed, which have been further proved in animal tumor model by TEM, immunohisto chemistry (IHC) and WB (Figure S29-31). Figure 5 shows flow cytometry analysis of autophagic flux in MCF-7 cells after drug exposure with NPG, Gsp, G-PEG-PLA, NPC and PTX at $50 \mathrm{nM}$ for 12 and $24 \mathrm{~h}$. Notably, NPG induced the formation of autophagic vesicles and autophagosomes more significantly than Gsp, G-PEGPLA, and NPC compared with the control groups, there is no significant change for parent PTX. Autophagy induced by NPG occurred within hours and was sustained up to $48 \mathrm{~h}$, a longer time period than those witnessed in Gsp, G-PEG-PLA, and NPC. Within the first two hours, there showed $11.8 \%$ autophagic vacuoles and autophagic flux for NPG treated cells compared with $4.1 \%$ in Ctrl groups, the groups averaged 11.9, 12.7, 11.2 and 5.5\% for Gsp, G-PEG-PLA, NPC, PTX respectively, after drug exposure for 12 and $24 \mathrm{~h}$, at $48 \mathrm{~h}$, most of the cells are in the late stage. 

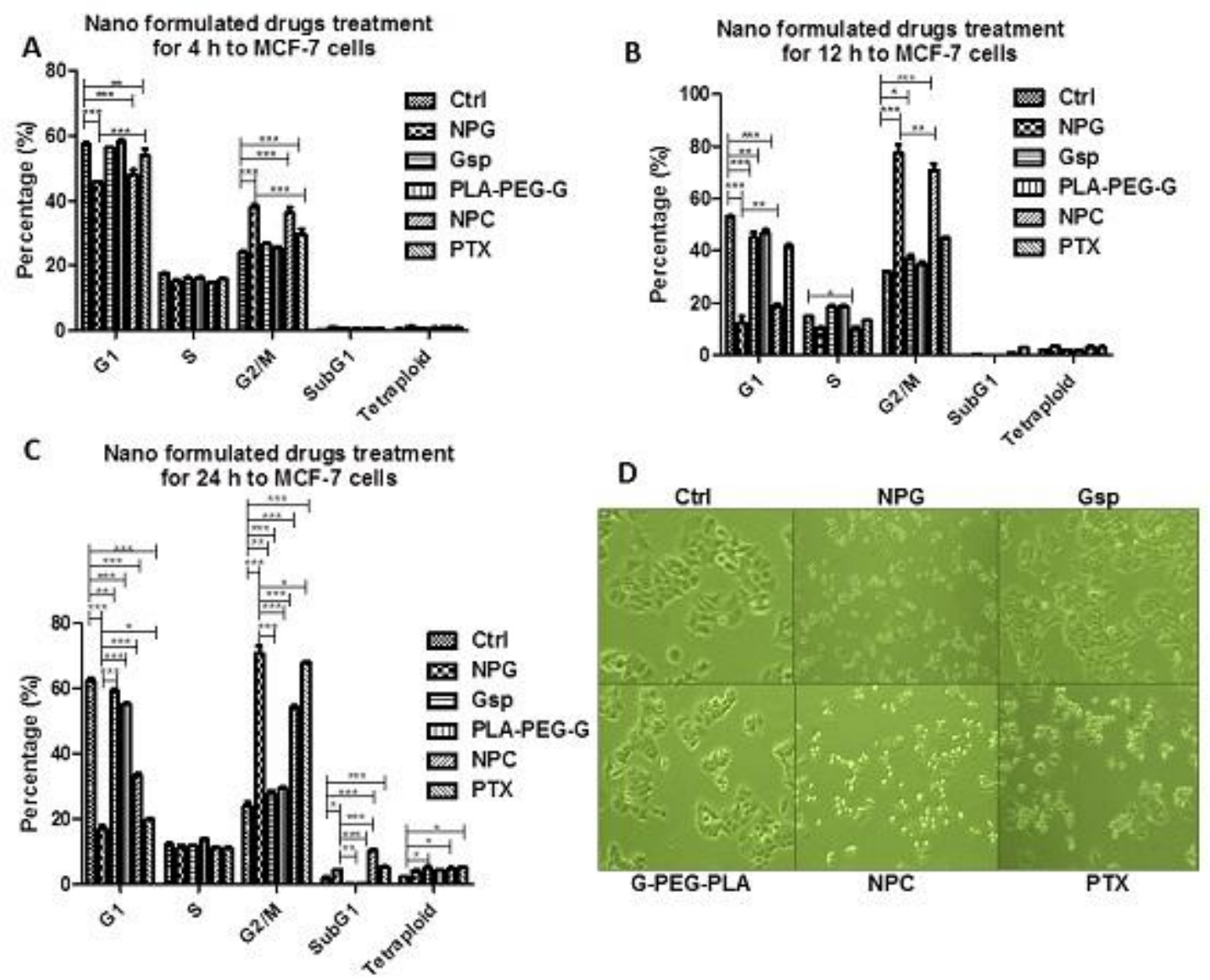

Figure 5: (A-C) FACS analysis of cell cycle distribution of MCF-7 cells after treatment with varying formulated of nanoparticles at $50 \mathrm{nM}$ for 4, 12 and $24 \mathrm{~h}$ Values of SD were less than 5\%.The histogram depicting the percentage of cells G1, S, G2/M, sub G1 phase; (D) The cellular morphology of the MCF-7 cells after the drug exposure under inverted phase microscope.

It has been reported that PTX causes inhibition of autophagy in breast cancer cells, both by decreasing autophagosome formation and by altering autophagosome trafficking and localization. ${ }^{25}$ Effective autophagy enhancement was observed in MCF-7 cells indicating that NPG stimulates cell death in the appropriate context. NPG induced robust autophagy while paclitaxel released from the micelles blocked a latter step in the pathway and autophagic flux by confocal microscopy. Autophagic vacuole accumulation and flux are both detected by Cyto-ID® Autophagy Green dye as observed after induction with NPG, Gsp, G-PEG-PLA, NPC and PTX for $12 \mathrm{~h}$ at $37^{\circ} \mathrm{C}$. White arrows indicate the characteristic punctate pattern of autophagosome, which occurs upon autophagic induction. leading to an accumulation of autophagosomes prior to induction of apoptosis though their effect on autophagy trafficking, resulting in eventual cell death, which suggested that manipulation of autophagy of nanotherapeutics may represent a therapeutic approach for improving breast cancer treatment options. It has been well demonstrated that autophagy depends on Atg5/Atg7, ${ }^{26}$ which is associated with microtubule-associated LC3I/II truncation and lipidation, NPG increased LC3 level by $22.9 \%$ and $32.2 \%$ for LC3I and LC3II separately at $8 \mathrm{~h}$, and $8.1 \%$ and $24 \%$ at $12 \mathrm{~h}$, and the autophagosome number in MCF-7 cells suggesting that the components in NPG act synergistically to induce autophagy-related cell death in breast cancer cells. Additionally, TEM microimages of MCF-7 cells induced by NPG were captured at early to late stage autophagy. (Figure 7, S7) The typical autophagic features of cells were observed after treatment with NPG, whereas untreated cells had normal nuclear and cytoplasmic morphology based on the TEM measurement. The initiation, elongation, closure, maturation and degradation stages involved in the autophagy process have been successfully captured separately. The control groups are shown in Figure 7A, without the induction of the drug, the cells are dormant and in an inactive state, clear nuclei and cytoplasma have been observed. The very initial step of autophagy is presented in Figure $7 \mathrm{~B}$, the surrounding and sequestering of cytoplasmic organelles and proteins within an isolation membrane, termed phagophore was observed with typical horse-shoe shape (as the red arrow shows). The nascent membranes are fused at their edges to form double-membrane vesicle-autophagosomes (as purple arrows show). Autophagosomes undergo a stepwise maturation process, including docking and fusion with acidified endosomal and/or lysosomal vesicles (as orange arrows show) eventually leading to the delivery of cytoplasmic contents to lysosomal components, where they fuse, then degrade. Furthermore, the time course of upstream regulated proteins associated with autophagy has also been examined (Figure S18). The treatment for NPG caused the down regulation of Akt and phosphorylated PI3K at 
Tyr199 by $35.6 \%$ and $32.2 \%$. Importantly, the downstream-phosphorylated mTOR at Ser248 revealed a $28.2 \%$ decrease. Inhibition of the upstream event such as mTOR phosphorylation and PI3k/Akt phosphorylation has been detected along with LC3II generation. Additionally, Beclin-1, also known as the autophagy-related gene, was up-regulated by $19.1 \%$ within $8 \mathrm{~h}$ in the time course study and reduced by $27.1 \%$ subsequently at the $24 \mathrm{~h}$ time point, it is most abundant at $24 \mathrm{~h}$ for the PTX group. A $29.6 \%$ decline in phosphorylated AMPK $\alpha$ at Th172 was also observed.
Moreover, significantly increased expression levels of Erk1 (p42 MAPK) and phosphorylation at Th202 (p-p42 MAPK) have also been recorded which are factors that contribute to autophagy stimulated by NPG. For NPG, G-PEG-PLA, NPC and PTX treated groups, p42 MAPK expression level shows $84.1 \%, 19.8 \%, 42.3 \%, 32.5 \%$ increases in comparison to the control groups respectively, and phosphorylated p42 MAPK increases by $111.2 \%, 221.8 \%, 249.7 \%, 222.7 \%$ respectively. While Gsp treated groups show comparable amounts of Erk1/2 stimulation to the control groups.
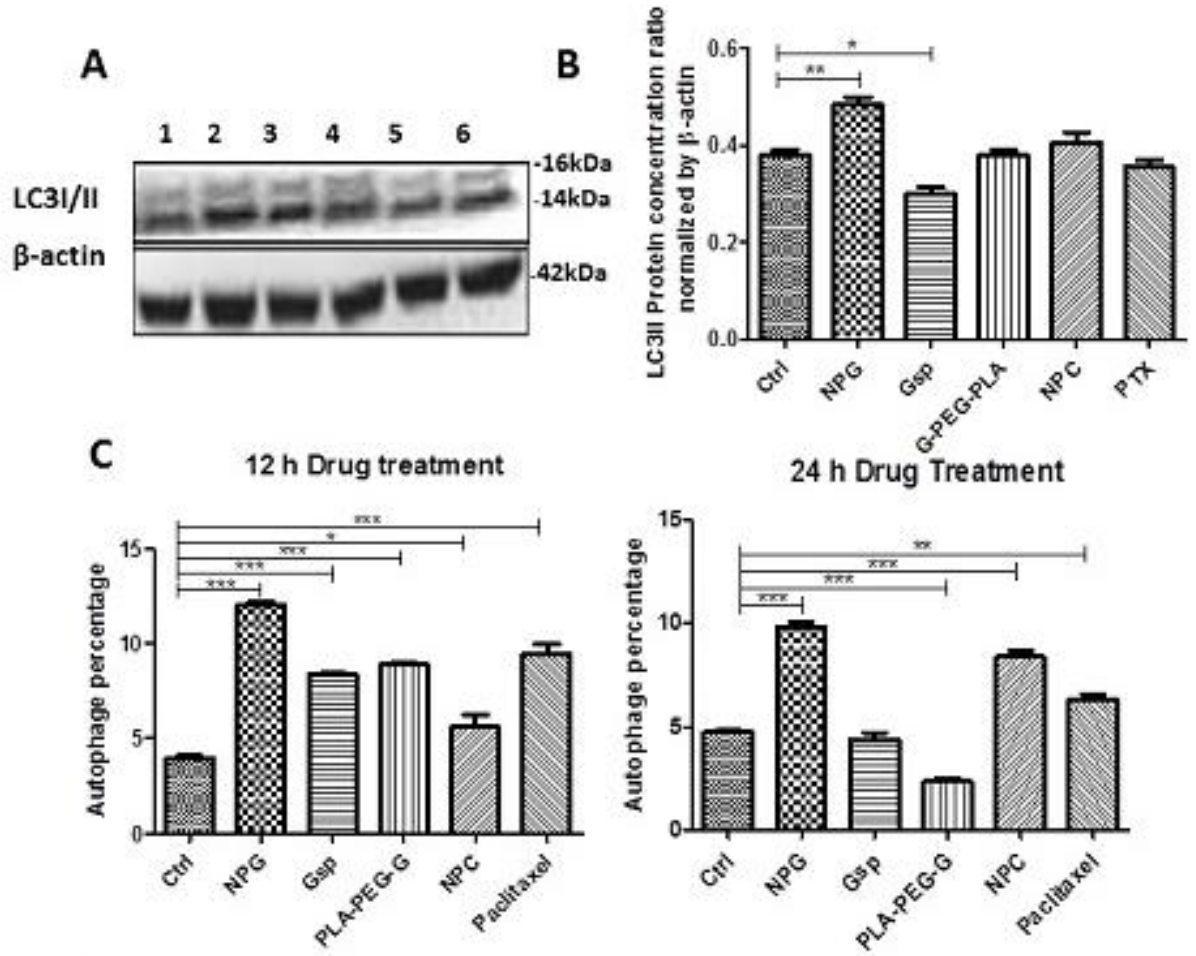

$24 \mathrm{~h}$ Drug Treatment
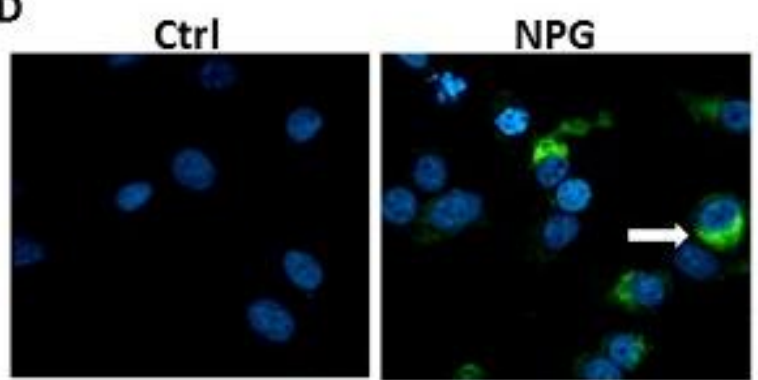

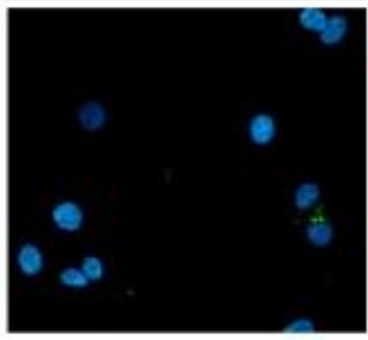

G-PEG-PLA

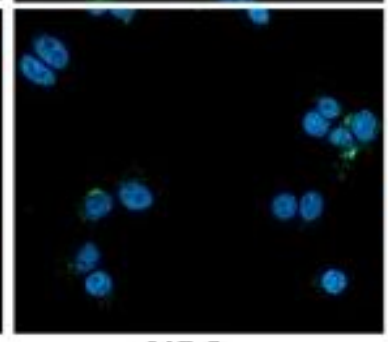

NPC
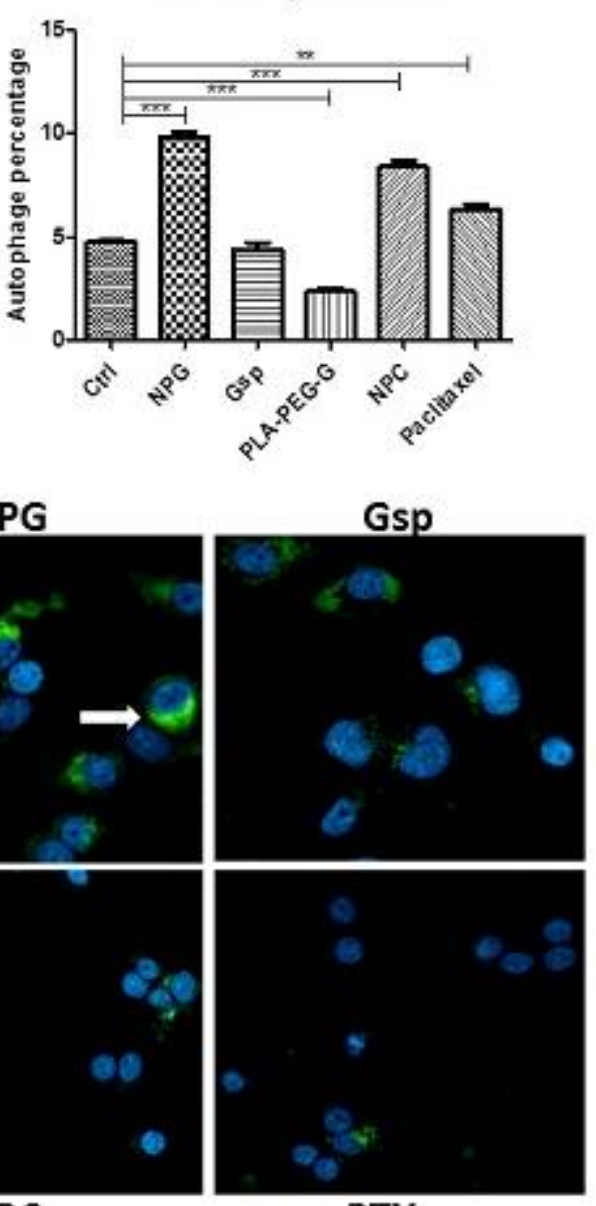

Figure 6: NPG induces autophagy in MCF-7 cells. (A) Western blot was used to detect the expression of LC3I/II. Cells were treated with $50 \mathrm{nM}$ NPG, G, G-PEG-PLA, NPC and PTX at $50 \mathrm{nM}$ for $12 \mathrm{~h}$. (B) Quantitative analysis of expression of LC3I/II based on WB. (C) Flow cytometry analysis of autophagic flux in MCF-7 cells detected by CytoID® Autophagy Green dye incubated with NPG, G, G-PEG-PLA, NPC and PTX at $50 \mathrm{nM}$ for 12, and 24 h. (D) Visualization of autophagic accumulation and autophagic flux by confocal microscopy. Autophagic vacuole accumulation and flux are both detected by Cyto-ID® Autophagyy Green dye as observed after induction with NPG, Gsp, G-PEG-PLA, NPC and PTX for $12 \mathrm{~h}$ at $37^{\circ} \mathrm{C}$. White arrows indicate the characteristic punctate pattern of autophagosome, which occurs upon autophagic induction. 

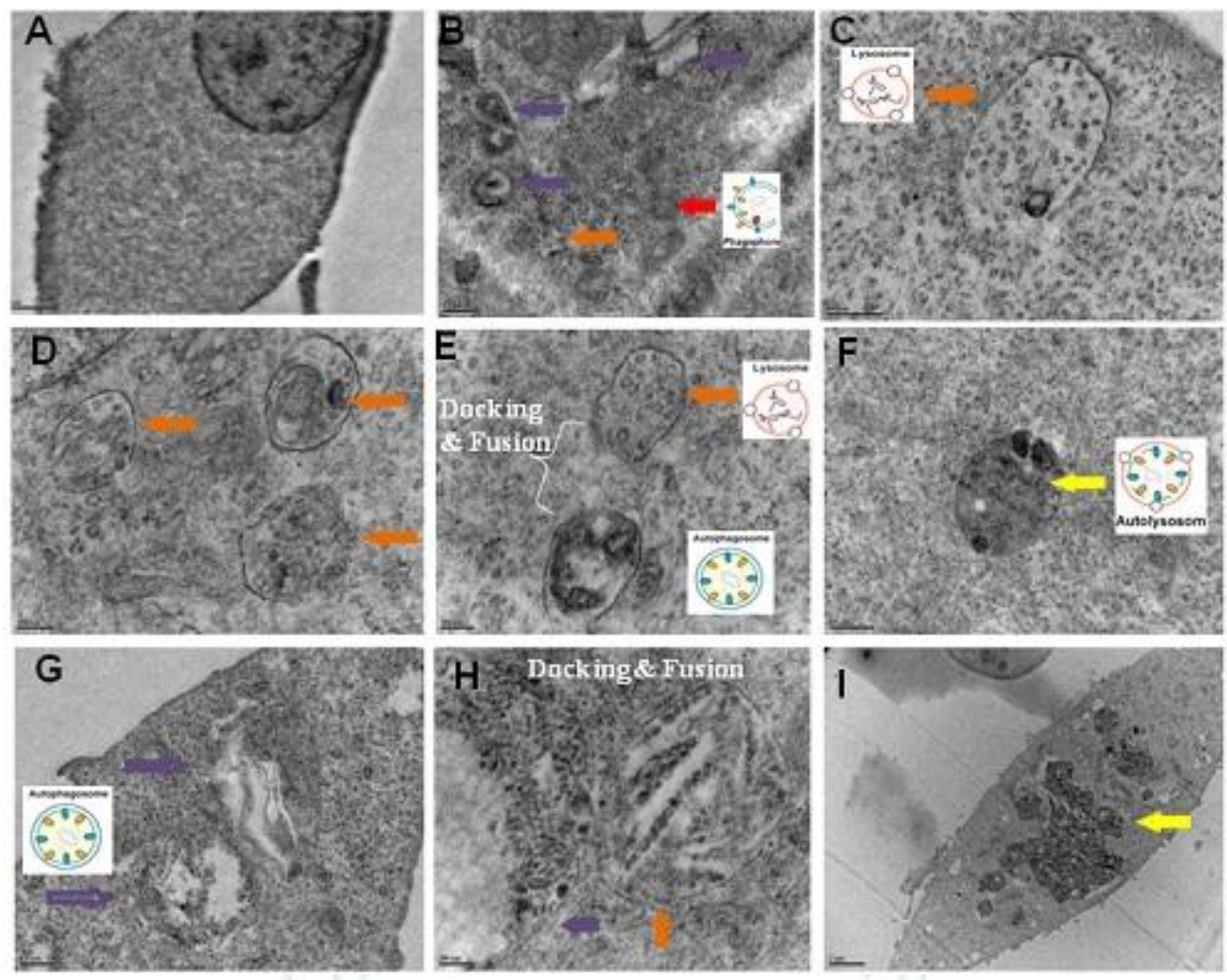

Figure 7: Electron microscopic analysis of autophagy in progressive stages. (A): Control samples without drug treatment. (B-I): NPG were incubated for $12 \mathrm{~h}$ at $50 \mathrm{nM}$. The cells were then fixed and processed for electron microscopy. B: Representative electron micrographs showing horseshoe shaped early stage of autophagy-phagophore (red arrow); C-D: Active lysosome (Orange arrows) which engulfs micro vesicles and vesicles; E-F: Representative graphs showing close contact of autophagosome (Purple arrows) with lysosome right before docking and fusion of autophagosome with lysosome; H-I: Selected micrographs showing the autolysosome and late stage of autophagy, the coalition of autolysosomes and lamella structure formed (Yellow arrows).

In regards to PTEN, there is no significant change observed for all the drug tested groups. The results proved that $\mathrm{PI} 3 \mathrm{k} / \mathrm{Akt} / \mathrm{mTOR}$ and ERK1/2 signaling pathways involved autophagic activity is another major cause of cell death besides mitotic arrest and augments the action of the encapsulated PTX. At the same time, few apoptotic nuclei from the TEM images have been observed unexpectedly. Moreover, according to the detection of the less than $5 \%$ apoptotic cell population shown in Figure S20-S21, it has been concluded that apoptotic activities induced by NPG play a minor role in tumor cell death.

\section{In Vivo Therapeutic Studies in Nude Mice Guide by} Proteomics Investigation

Finally, the anti-tumor activity has been evaluated in vivo. Athymic nude mice were implanted subcutaneously with MCF cells. When the animals developed palpable tumors after 10 days, they were treated intravenously with NPG and PTX with equal equivalent $(2.36 \mathrm{mg}$ PTX $/ \mathrm{kg}$ body weight in a total volume of $100 \mu \mathrm{L}$ ) once a week for sequential 5 weeks. Animals in the control group received intravenous administration (i. v.) of saline. In vivo drug distribution within $48 \mathrm{~h}$ are shown in Figure 8 in which NPG treated group were injected with the NPG containing 98\% PTX and 2\% PTX-oregon green 488 conjugate, and PTX treated group using 98\% PTX and 2\% PTX-oregon green 488 conjugate PTX mixture. Mice in the NPG treated group display dramatically elevated drug uptake in tumor xenograft tissue as compared to Mice of the PTX group which shows nonspecific drug distribution. In addition, the drug excretion though urine was recorded and made detectable through red fluorescence in mice bladders. NPG shows double extended circulation half life time. The first fluorescent urine was recorded at $6 \mathrm{~h}$ for the mice in PTX group, while it was at $12 \mathrm{~h}$ after the NPG administration. Tumor growth curve, mice body weight change, representative images of gross $\mathrm{MCF}-7$ cell tumors and tumor metastasis detection from dissected organs from the nude mice treated with either control or PTX and NPG respectively are shown in Figure 9. Mice in the control group developed rapidly growing subcutaneous tumors. After five injections, the average tumor volumes in Ctrl, PTX and NPG treatment groups were $3883.58,1868.57$ and $1084.93 \mathrm{~mm}^{3}$ respectively. Remarkably, animals in the NPG group exhibited significantly retarded tumor development compared to the animals in the control group $(* \mathrm{p}<0.05)$, and shows more potent retarded tumor development than the PTX group. 


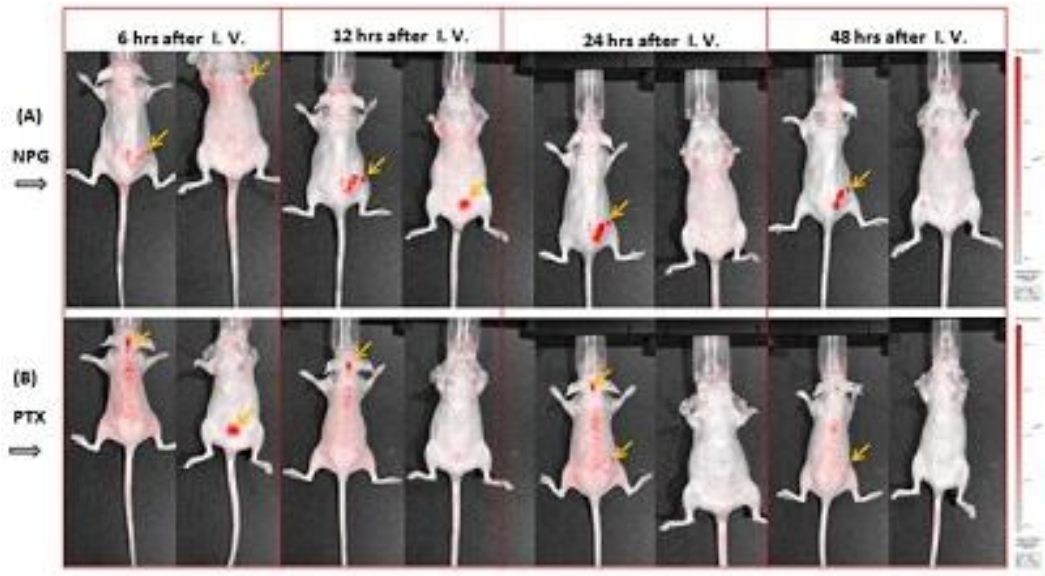

Figure 8 In vivo drug distributions of NPG and PTX in MCF-7 tumor-bearing mice. The NPG was encapsulated with 98\% PTX and 2\% PTX-Oregon Green 488 Conjugate; PTX groups use 98\% PTX and 2\% PTX-Oregon Green 488 conjugate. The drug was injected i. v. into MCF-7 cells xenograft nude mice. (A) Imaging of mice at 6, 12, 24, and 48 h after injection for NPG group. (B) Imaging of mice at 6, 12, 24, and $48 \mathrm{~h}$ after injection for PTX group. (Exposure time=1s, Ex=465 nm, Em=520 nm)

A

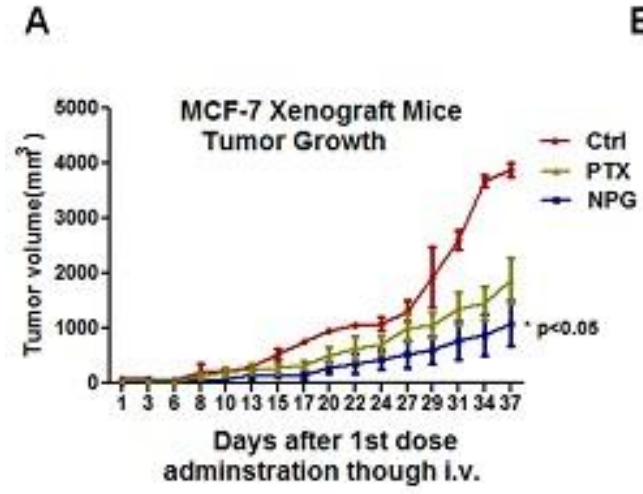

c

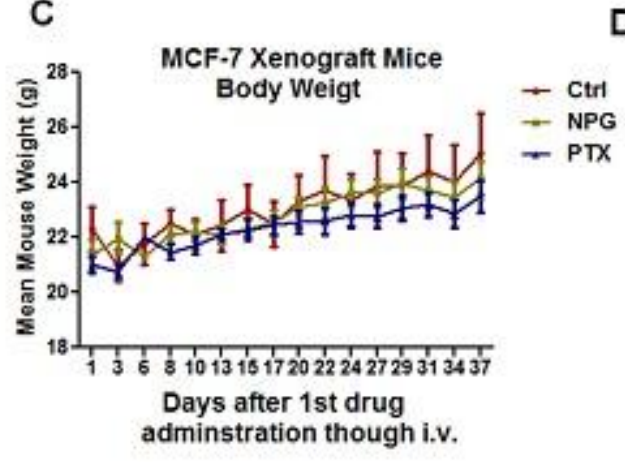

B

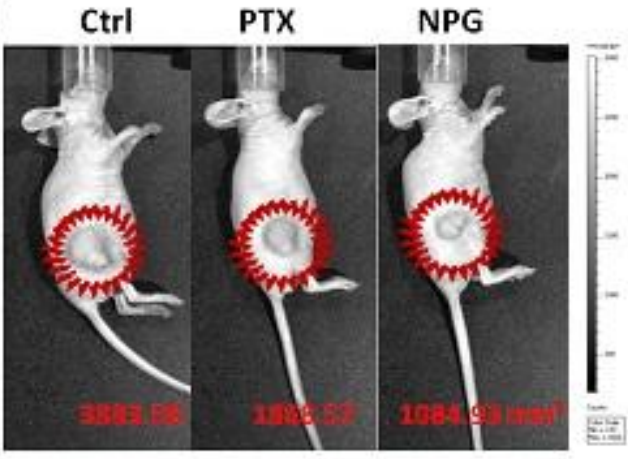

D

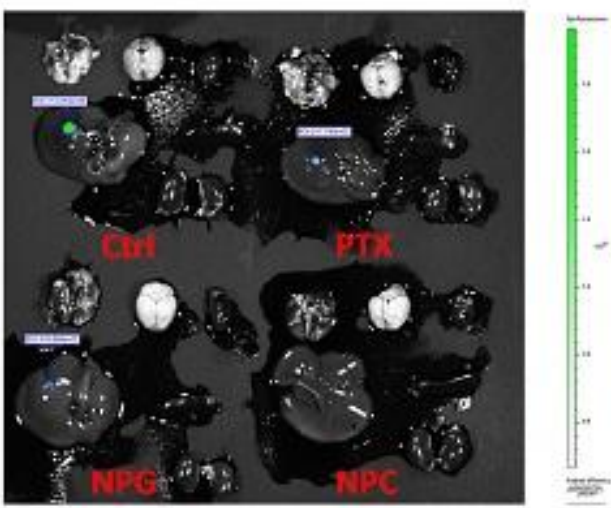

Figure 9: Antitumor effect of NPG nanoparticle in the breast tumor xenografts. (A) The growth curve of MCF-7 xenografts showed that NPG significantly inhibited the growth of tumor as compared with the control $(* \mathrm{p}=0.028)$, and retard tumor development more potent than PTX group. (B) The body weights of the mice in all groups (C) Typical photos of mice on day 35. After five injections, the average tumor volumes in Ctrl, PTX and NPG treatment groups were 3883.58, 1868.57, $1084.93 \mathrm{~mm} 3$ respectively. (D) Representative GFP fluorescence images of organs dissected from control, PTX and NPG-treated mice to evaluate the tumor metastasis. The organs are presented as lung, brain, heart, liver, spleen and kidney in an order from left to the right and from top to the bottom.

The body weight of the mice in the NPG, PTX and control group shows no significant change. Liver metastasis was detected though bio-fluorescence imaging of the dissected organs from the nude mice injected with GFP transfected MCF-7 tumor cells (Figure 9D, Figure S24). Blue circles indicate the tumor metastasis nodules, and the fluorescence intensity was quantitated by the IVIS spectrum. It revealed that NPG treatment inhibited liver metastasis from breast tumors as well as PTX with the tumor metastasis inhibition rate of $77.83 \%$ and $70.71 \%$ respectively according to the quantitation of photointensity of the GFP expression site. Notably, NPG markedly alleviated the vein injury traditionally caused by PTX as shown in Figure S25, 
hematoxylin and eosin (H\&E) stained micrographs of the blood vessel of the mice tail vein from PTX treated group showed deformed and narrow vein compared with veins the normal morphology after NPG treatment. The vasculature photographs revealed that the vessels of the mice in PTX group are dilated, tortuous, and hyperpermeable, in contrast, the vascular architecture maintained clear, orderly characteristics for the vascular walls in the NPG treated mice which indicated practical importance for potential clinic implementation.

The whole blood hematologic and serum biochemical variables were also evaluated for the mice (Figure S26). The complete blood count (CBC) revealed a slightly decreased leukocytes density for the NPG group. Importantly, high platelet counts from the control group, considered to be one indicator of cancer, was ameliorated to normal reference range for $100 \%$ of the mice in the NPG treated group, in comparison to $40 \%$ of the PTX group. Hematology serum biochemistry parameters show that the serum blood urea nitrogen (BUN) and creatinine level in the control mice was 19.0 $\mathrm{mg} / \mathrm{dL}(20.0-26.0 \mathrm{mg} / \mathrm{dL}$ for the normal range) and 0.3 $\mathrm{mg} / \mathrm{dL}(0.5-1.6 \mathrm{mg} / \mathrm{dL})$ in average which is lower than the normal range, the serum BUN and creatinine levels in the NPG treated mice were varied within 20.9 to 24.0 $\mathrm{mg} / \mathrm{dL}$ which is elevated to the normal range standard reference. There is no remarkable change for the serum levels of alanine amino transferase (ALT) and alkaline phosphatase (ALP) in all of the mice.

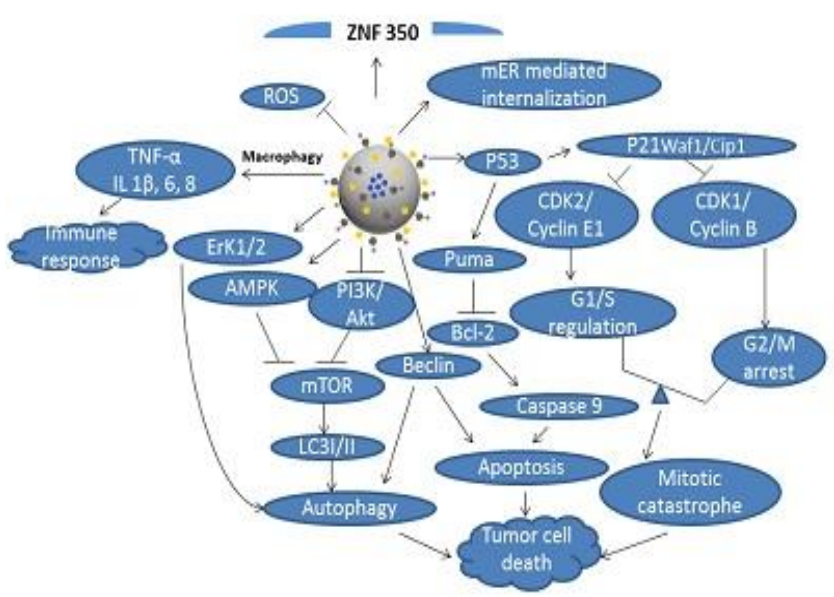

Figure 10: Summary of the validated pathway induced by NPG

Furthermore, flow cytometric assay of drug-mediated immune response in peripheral-blood cells of the mice was conducted (Figure S27). The cells were subsequently permeabilized and stained with cellsurface markers. The lymphocytes including CD19 (+) B cell and CD49b (+) Natural Killer (NK) cells in blood were not significantly affected.

WB and immunohistochemistry (IHC) analysis in vivo revealed upregulated autophagy indicator-LC3 expression levels in NPG treated mice in comparison to the control mice.

The H\&E stained and the TEM microimages from the tumor sections of the mice treated by the nanoparticles NPG, PTX and saline are shown in Figure S28 and S29, catastrophic cytoplasmic vacuolization has been observed in the tumor tissue of the nanoparticle treated mice more obviously than the other group due to the proposed autophagy caused by NPG.

The proteomics annotation of NPG response in breast cancer cells reveals that the ZNF350 are stimulated during treatment, $\mathrm{WB}$ and $\mathrm{IHC}$ analysis in vivo confirmed the proteomic data demonstrating that the top gene ZNF350 (or ZBRK1) was significantly more abundant in NPG treated mice than in the control and PTX group (Figure S30,31). ZNF350 is considered as valuable anti-oncogene for NPG anti-tumor activity in breast cancer. Importantly, activation of ZNF350 is absent in the annotation from the proteomics profiling for all other single constitutes formulated in the nanoparticle as Gsp, polymeric gossypol and PTX. It revealed that ZNF 350 is stimulated uniquely by the integrated drug interaction formulated in the nanoparticles other than the single drug regime. Meanwhile the drug synergism in NPG enhanced the overall therapeutic effect. Therefore our data strengthen the conclusion that ZNF350 may acts as an oncosuppressor in the presence of NPG, revealing ZNF350 to be as a promising therapeutic gene from the antitumor study in vitro and in vivo based on the finding from nanoparticulated drugs. The summary of the validated pathway induced by NPG is shown in Figure 10. NPG causes comprehensive intracellular signal transduction after the mER facilitated drug internalization and digestion (Supp Info). NPG induces breast tumor cell death though mitotic catastrophe with synergism from PTX, and induces unexpected autophagy resulting from the combinational composites of NPG which is distinct from the single drug actions.

\section{CONCLUSIONS}

In summary, we successfully synthesized a novel estrogen anchored multifunctional polymeric nanoparticle NPG for the codelivery of a mitotic agent paclitaxel and a BH3 mimic gossypol which shows advantages over the conventional paclitaxel formulation in vitro and in vivo. The drug mechanism was investigated assisted by systematic SILAC based proteomic study. The NPG treatment in vivo showed improved drug hydrophilicity to avoid the solvent associated side effect of Taxol in clinical use, targeted tumor accumulation, increased drug uptake, extended circulation time in vivo, significant metastasis inhibition, enhanced anti-tumor therapeutic efficacy, protection from vein injury due to intravenous administration, and negligible damage to kidney, liver function as well as immune system at given dosage. Systematic proteomics study and validation in vitro and in vivo for drug response of NPG in breast cancer revealed that synergistically enhanced G2/M phase arrest, PI3K/Akt/mTOR and Erk1/2 mediated autophagy mainly contribute to the mechanism of cancer cell death, instead of the expected inhibition of pro-apoptotic BcL2 from gossypol besides the mitotic catastrophe resulting from paclitaxel which shows that multifunctional nanoparticles stimulate unique cellular response and results in new mechanisms compared with single drug regimes. NPG is evident as a promising drug 
candidate for clinical translation to treat mER (+) tumors. Proteomics data and the validation suggest that the upregulation of ZNF350 induced by the combinational NPG may be associated with the antitumor efficacy against breast cancer in vivo, thereby ZNF350 as a new therapeutic target reveals the treatment paradigms for the combinational therapeutic nanoparticles and it is worthwhile for further investigation. In a word, utilization of proteomics for the investigation of mechanic pathways triggered by multifunctional nanoparticles for cancer therapy provides critical insight in nanodrug development and their therapeutic targets.

\section{ASSOCIATED CONTENT}

Supporting information available: Experimental details; Methods; NMR, MS spectra; Solubility test; DLS; WB; IHC; TEM; SEM; H\&E stained images; SRB assay; Tumor colony inhibition assay; Proteomics files; Hematology; Flow cytometry for immune response in vivo. This material is available free of charge via the Internet at http://pubs.acs.org.

\section{REFERENCES}

1 Stratton MR, Campbell PJ, Futreal PA, Nature. 2009; 458:719-724

2 Hu CM, Aryal S, Zhang L, Ther Deliv. 2010; 1:323-34.

3 Liao L, Liu J, Dreaden EC, Morton SW, Shopsowitz KE, Hammond PT, Johnson JA, Journal of the American Chemical Society. 2014; 136:5896-5899

4 Huang P, Wang D, Su Y, Huang W, Zhou Y, Cui D, Zhu X, Yan D, Journal of the American Chemical Society. 2014; 136:11748-11756

5 Markovsky E, Baabur-Cohen H, Satchi-Fainaro R, Journal of Controlled Release. 2014; 187:145-157.

$6 \quad$ Ma L, Kohli M, Smith A, Acs Nano. 2013; 7:9518-9525.

7 Manzoor AA, Lindner LLH, Landon CD, Park JY, Simnick AJ, Dreher MR, Das S, Hanna G, Park W, Chilkoti A, Koning GA, Ten Hagen TL, Needham D, Dewhirst MW, Cancer Res. 2012; 72:5566-75.

8 Ong SE, Blagoev B, Kratchmarova I, Kristensen DB, Steen H, Pandey A, Mann M, Molecular \& Cellular Proteomics. 2002; 1:376-386.

9 Ong SE, Mann M, Nat Chem Biol. 2005; 1:252-262.

10 Verano-Braga T, Miethling-Graff R, Wojdyla K, RogowskaWrzesinska A, Brewer JR, Erdmann H, Kjeldsen F, ACS Nano. 2014; 8:2161-2175.

11 Hofmann D, Tenzer S, Bannwarth MB, Messerschmidt C, Glaser S-F, Schild H, Landfester K, Mailänder V, ACS Nano. 2014; 8:10077-10088

12 Kelly MJ, Levin ER, Trends Endocrinol Metab. 2001; 12:152-6

13 Chaudhri RA, Olivares-Navarrete R, Cuenca N, Hadadi A, Boyan BD, Schwartz Z, J Biol Chem. 2012; 287:7169-81
Conflict of interest: The authors declare no competing final interest.

\section{ACKNOWLEDGMENT}

The authors are grateful for the support from the National Natural Science Foundation of China (Grant No. 81372383), and the startup fund from College of Pharmacy, University of South Florida; This work has been supported in part by the Florida Center of Excellence for Drug Discovery \& Innovation at the University of South Florida; The authors thank Drs. S. Karoly and A. Garces at the Lisa Muma Weitz Advanced Microscopy Core Laboratory for the support in data acquisition of flow cytometry, small animal imaging, and electronic microscopy, Dr. R. Sprunt at Moffitt Cancer Center for the Maldi-Tof mass spectroscopy, we thank A. Lindon and B. Karen at College of Medicine Vivarium, the Division of Comparative Medicine, University of South Florida; We thank M. Torres and T. Casagni at Comparative Medicine, University of South Florida for the CBC and biochemical analysis. We also thank A. Georgia at USF Health Diagnostic Laboratory for the tissue processing.

14 Li L, Haynes MP, Bender JR, Proc Natl Acad Sci U S A. 2003; 100:4807-12

15 M. Kampa, G. Notas, V. Pelekanou, M. Troullinaki, M. Andrianaki, K. Azariadis, E. Kampouri, K. Lavrentaki, and E. Castanas, Steroids. 2012; 77:959-67

16 Micevych PE, Kelly MJ, Neuroendocrinology. 2012; 96:10310

17 Jordan MA, Wilson L, Nature Reviews Cancer. 2004; 4:253265

18 Oliver CL, Bauer JA, Wolter KG, Clinical Cancer Research. 2005; 11:5659-5659

19 Yin JJ, Sharma S, Shumyak SP, Wang ZX, Zhou ZW, Zhang Y, Guo P, Li CZ, Janwar JR, Yang T, Mohapatra SS, Liu W, Duan W, Wang JC, Li Q, Zhang X, Tan J, Jia L, Liang J, Wei MQ, Li X, Zhou SF, PLoS One. 2013; 8:e62289

20 Torchilin VP, Pharmaceutical Research. 2007; 24:1-16

21 Lin L-F, Chuang C-H, Li C-F, Liao C-C, Cheng C-P, Cheng T-L, Shen M-R, Tseng JT, Chang W-C, Lee W-H, Wang J-M, Cancer Research. 2010; 70:192-201

22 Rutter JL, Smith AM, Davila MR, Sigurdson AJ, Giusti RM, Pineda MA, Doody MM, M. Tucker A, Greene MH, Zhang JH, Struewing JP, Human Mutation. 2003; 22:121-128

23 Swami M, Nat Rev Cancer. 2010; 10:597-597.

24 Cox J, Mann M, Nat Biotechnol. 2008; 26:1367-72

25 Veldhoen RA, Banman SL, Hemmerling DR, Odsen R, Simmen T, Simmonds AJ, Underhill DA, Goping IS, Oncogene. 2013; 32:736-746

26 Kang R, Zeh HJ, Lotze MT, Tang D, Cell Death and Differentiation. 2011; 18:571-580 